\title{
An inductive method for proving the transcendence of certain series
}

\author{
by
}

Daniel Duverney (Lille) and Kumiko Nishioka (Yokohama)

1. Introduction. If $\alpha$ is an algebraic number, we denote by $\alpha$ the maximum of the absolute values of the conjugates of $\alpha$ and by $\operatorname{den}(\alpha)$ the least positive integer such that $\operatorname{den}(\alpha) \alpha$ is an algebraic integer, and we set $\|\alpha\|=\max \{\alpha, \operatorname{den}(\alpha)\}$. Then for nonzero algebraic $\alpha$, we have the fundamental inequalities

$$
|\alpha| \geq\|\alpha\|^{-2[\mathbb{Q}(\alpha): \mathbb{Q}]} \quad \text { and } \quad\left\|\alpha^{-1}\right\| \leq\|\alpha\|^{2[\mathbb{Q}(\alpha): \mathbb{Q}]}
$$

(cf. [12, Lemma 2.10.2]).

Let $K$ be an algebraic number field and $O_{K}$ be the ring of integers in $K$. Let $r$ and $L$ be integers such that $r \geq 2$ and $L \geq 1$. We consider the function

$$
\Phi_{0}(x)=\sum_{k=0}^{\infty} \frac{E_{k}\left(x^{r^{k}}\right)}{F_{k}\left(x^{r^{k}}\right)},
$$

where

$$
\begin{gathered}
E_{k}(x)=a_{k 1} x+a_{k 2} x^{2}+\ldots+a_{k L} x^{L} \in K[x], \\
F_{k}(x)=1+b_{k 1} x+b_{k 2} x^{2}+\ldots+b_{k L} x^{L} \in O_{K}[x], \\
\log \left\|a_{k l}\right\|, \log \left\|b_{k l}\right\|=o\left(r^{k}\right), \quad 1 \leq l \leq L .
\end{gathered}
$$

The aim of this paper is to study the arithmetical nature of $\Phi_{0}(\alpha)$ when $\alpha \in K, 0<|\alpha|<1$, and $F_{k}\left(\alpha^{r^{k}}\right) \neq 0$ for every $k \geq 0$.

It should be noticed that in some cases $\Phi_{0}(x)$ can be explicitly computed as a rational function. Specific examples are, with $r=2$ :

2000 Mathematics Subject Classification: Primary 11J81.

Key words and phrases: transcendence, Mahler's method. 


$$
\begin{aligned}
\sum_{k=0}^{\infty} \frac{x^{2^{k}}}{1-x^{2^{k+1}}} & =\frac{x}{1-x}, \\
\sum_{k=0}^{\infty} \frac{2^{k} x^{2^{k}}}{1+x^{2^{k}}} & =\frac{x}{1-x} \\
\sum_{k=0}^{\infty} \frac{(-2)^{k} x^{2^{k}}}{x^{2^{k+1}}-x^{2^{k}}+1} & =\frac{x}{x^{2}+x+1} .
\end{aligned}
$$

The first equality is due to Lucas [9]. The latter two equalities are proved in Duverney [4] but are evidently older. In the case where $r=3$, we have for example

$$
\sum_{k=0}^{\infty} \frac{3^{k} x^{3^{k}}\left(1-x^{2 \cdot 3^{k}}\right)}{x^{4 \cdot 3^{k}}+x^{2 \cdot 3^{k}}+1}=\frac{x}{1-x^{2}} .
$$

This equality is proved in Duverney and Shiokawa [7]. Clearly for these examples, $\Phi_{0}(\alpha) \in K$ if $\alpha \in K$.

Our main result will be

Transcendence CRIterion. $\Phi_{0}(\alpha)$ is algebraic if and only if $\Phi_{0}(x)$ is a rational function.

In fact we will prove the more precise Theorem 6 below (see Section 3 ), which will also give us a way of proving that $\Phi_{0}(x) \notin K(x)$. The proof of Theorem 6 (and therefore of the transcendence criterion) relies on Mahler's transcendence method, more precisely on the following result, which is a special case of a theorem of Loxton and van der Poorten [8] (cf. [12, Theorem 2.9.1]).

THEOREM 1. Let $K$ be an algebraic number field, $r \geq 2$ be an integer, $\left\{\Phi_{n}(x)\right\}_{n \geq 0}$ be a sequence in the ring of formal power series $K[[x]]$ and $\alpha \in K$ with $0<|\alpha|<1$. If the following three properties are satisfied, then $\Phi_{0}(\alpha)$ is transcendental. $=O\left(r^{n}\right)$.

(I) $\Phi_{n}\left(\alpha^{r^{n}}\right)=a_{n} \Phi_{0}(\alpha)+b_{n}$, where $a_{n}, b_{n} \in K$, and $\log \left\|a_{n}\right\|, \log \left\|b_{n}\right\|$

(II) If $\Phi_{n}(x)=\sum_{l=0}^{\infty} \sigma_{l}^{(n)} x^{l}$, then for any $\varepsilon>0$ there is a positive integer $n_{0}$ such that

$$
\log \left\|\sigma_{l}^{(n)}\right\| \leq \varepsilon r^{n}(1+l)
$$

for any $n \geq n_{0}$ and $l \geq 0$.

(III) Let $\left\{s_{l}\right\}_{l \geq 0}$ be variables and

$$
F(x ; s)=F\left(x ;\left\{s_{l}\right\}_{l \geq 0}\right)=\sum_{l=0}^{\infty} s_{l} x^{l},
$$


in such a way that

$$
F\left(x ; \sigma^{(n)}\right)=F\left(x ;\left\{\sigma_{l}^{(n)}\right\}_{l \geq 0}\right)=\Phi_{n}(x) .
$$

Then for any polynomials $P_{0}(x, s), \ldots, P_{d}(x, s) \in K\left[x,\left\{s_{l}\right\}_{l \geq 0}\right]$ and

$$
E(x, s)=\sum_{j=0}^{d} P_{j}(x, s) F(x ; s)^{j},
$$

there is a positive integer $I$ with the following property: if $n$ is sufficiently large and $P_{0}\left(x, \sigma^{(n)}\right), \ldots, P_{d}\left(x, \sigma^{(n)}\right)$ are not all zero, then ord $E\left(x, \sigma^{(n)}\right)$ $\leq I$, where ord denotes the zero order at 0 .

However, applying Theorem 1 to $\Phi_{0}(x)$ will not be an easy task, because of condition (III). Thus the second section will be devoted to the proof of Theorem 3, in which condition (III) will be replaced by a simpler one, namely, some kind of irrationality measure of the function $\Phi_{0}(x)$. The tool in this section is an inductive method developed in Duverney [5].

Then, in the third section, we will use rather classical tools in approximation theory, in order to compute this irrationality measure. By introducing low-order Padé approximants of the functions $\Phi_{n}(x)$ connected to $\Phi_{0}(x)$ by the equality (41), we will arrive at Theorem 6, which implies the transcendence criterion and will enable us to obtain transcendence results. These results will be developed in Section 5 (see Theorems 7-11).

\section{An inductive method}

TheOREM 2. Let $K$ be an algebraic number field, $r$ and $L$ be integers such that $r \geq 2$ and $L \geq 1$, and

$$
S=\Phi_{0}(x)=\sum_{k=0}^{\infty} \frac{E_{k}\left(x^{r^{k}}\right)}{F_{k}\left(x^{r^{k}}\right)},
$$

where

$$
\begin{aligned}
& E_{k}(x)=a_{k 1} x+a_{k 2} x^{2}+\ldots+a_{k L} x^{L} \in K[x], \\
& F_{k}(x)=1+b_{k 1} x+b_{k 2} x^{2}+\ldots+b_{k L} x^{L} \in K[x] .
\end{aligned}
$$

Suppose that there is a positive constant $c_{1}$ such that for any $M \geq 1$ and any polynomials $A_{0}, A_{1} \in K[x]$, not both zero, satisfying $\operatorname{deg} A_{0}, \operatorname{deg} A_{1} \leq M$,

$$
\operatorname{ord}\left(A_{0}+A_{1} S\right) \leq c_{1} M \text {. }
$$

Then for any positive integer $d$ there is a positive constant $c_{d}$ such that for any $M \geq 1$ and any polynomials $A_{0}, A_{1}, \ldots, A_{d} \in K[x]$, not all zero, satisfying $\operatorname{deg} A_{i} \leq M, 0 \leq i \leq d$,

$$
\operatorname{ord}\left(A_{0}+A_{1} S+\ldots+A_{d} S^{d}\right) \leq c_{d} M
$$


Proof. Let

$$
\Phi_{n}(x)=\sum_{k=0}^{\infty} \frac{E_{n+k}\left(x^{r^{k}}\right)}{F_{n+k}\left(x^{r^{k}}\right)}, \quad R_{n}=\Phi_{n}\left(x^{r^{n}}\right), \quad T_{n}=\sum_{k=0}^{n-1} \frac{E_{k}\left(x^{r^{k}}\right)}{F_{k}\left(x^{r^{k}}\right)} .
$$

Then $S=T_{n}+R_{n}$. We prove (2) by induction on $d$. If $d=1$, then (2) is the same as (1). Suppose that for a given $d \geq 2$, we have

$$
\operatorname{ord}\left(B_{0}+B_{1} S+\ldots+B_{d-1} S^{d-1}\right) \leq c_{d-1} M
$$

for any $B_{0}, \ldots, B_{d-1} \in K[x]$, not all zero, with $\operatorname{deg} B_{i} \leq M, 0 \leq i \leq d-1$. We may assume $c_{d-1} \geq 1$ and $A_{d} \neq 0$. Let $e=d L$. For every $n>0$, there exist $Q_{n}(x) \in K[x]$ with $Q_{n}(x) \neq 0$, and $P_{n 1}(x), \ldots, P_{n d}(x) \in K[x]$ such that

$$
\begin{gathered}
\operatorname{deg} Q_{n} \leq d e, \quad \operatorname{deg} P_{n i} \leq d e, \quad 1 \leq i \leq d \\
Q_{n}(x) \Phi_{n}(x)^{i}-P_{n i}(x)=x^{d e+e+1} G_{n i}(x), \quad 1 \leq i \leq d,
\end{gathered}
$$

where

$$
G_{n i}(x)=\sum_{l=0}^{\infty} g_{n i l} x^{l} \in K[[x]] .
$$

For this we choose $Q_{n}(x)$ in such a way that the terms of degrees $d e+1$, $\ldots, d e+e$ vanish in the Taylor expansion of $Q_{n}(x) \Phi_{n}(x)^{i}$ for $i=1, \ldots, d$. We only have to solve a linear homogeneous system which has de equations and $d e+1$ unknowns.

LEMMA 1. ord $G_{n 1}(x) \leq \gamma$, where $\gamma=c_{1}(d e+L)-(d e+e+1)$.

Proof. In (4), replacing $x$ by $x^{r^{n}}$, we have

$$
Q_{n}\left(x^{r^{n}}\right)\left(S-T_{n}\right)-P_{n 1}\left(x^{r^{n}}\right)=x^{(d e+e+1) r^{n}} G_{n 1}\left(x^{r^{n}}\right) .
$$

Multiplying both sides by $D_{n}=\prod_{k=0}^{n-1} F_{k}\left(x^{r^{k}}\right)$, we have

$$
D_{n} Q_{n}\left(x^{r^{n}}\right) S-Q_{n}\left(x^{r^{n}}\right) D_{n} T_{n}-D_{n} P_{n 1}\left(x^{r^{n}}\right)=x^{(d e+e+1) r^{n}} D_{n} G_{n 1}\left(x^{r^{n}}\right) \text {. }
$$

Since $\operatorname{deg} D_{n}, \operatorname{deg} D_{n} T_{n} \leq L r^{n}$,

$$
\operatorname{deg} D_{n} Q_{n}\left(x^{r^{n}}\right), \operatorname{deg}\left(Q_{n}\left(x^{r^{n}}\right) D_{n} T_{n}+D_{n} P_{n 1}\left(x^{r^{n}}\right)\right) \leq(L+d e) r^{n} .
$$

By (1) we have

$$
\text { ord } G_{n 1}\left(x^{r^{n}}\right) \leq\left(c_{1}(d e+L)-(d e+e+1)\right) r^{n},
$$

which implies the lemma.

We define $P_{n 0}(x)=Q_{n}(x), G_{n 0}(x)=0$. In (4), replacing $x$ by $x^{r^{n}}$, we obtain, for every $i=0,1, \ldots, d$,

$$
Q_{n}\left(x^{r^{n}}\right)\left(S-T_{n}\right)^{i}-P_{n i}\left(x^{r^{n}}\right)=x^{(d e+e+1) r^{n}} G_{n i}\left(x^{r^{n}}\right) .
$$


We develop $\left(S-T_{n}\right)^{i}$ and write the equality (5) in matrix form. Then we get

$$
Q_{n}\left(x^{r^{n}}\right) \mathcal{M}_{n}\left(\begin{array}{c}
1 \\
S \\
\vdots \\
S^{d}
\end{array}\right)-\left(\begin{array}{c}
P_{n 0}\left(x^{r^{n}}\right) \\
P_{n 1}\left(x^{r^{n}}\right) \\
\vdots \\
P_{n d}\left(x^{r^{n}}\right)
\end{array}\right)=x^{(d e+e+1) r^{n}}\left(\begin{array}{c}
0 \\
G_{n 1}\left(x^{r^{n}}\right) \\
\vdots \\
G_{n d}\left(x^{r^{n}}\right)
\end{array}\right)
$$

where

$$
\mathcal{M}_{n}=\left(\begin{array}{cccc}
1 & 0 & \ldots & 0 \\
-T_{n} & 1 & \ldots & 0 \\
T_{n}^{2} & -2 T_{n} & \ldots & 0 \\
\ldots \ldots \ldots \ldots \ldots \ldots \ldots \ldots \ldots \ldots & \\
(-1)^{d} T_{n}^{d} & (-1)^{d-1}\left(\begin{array}{l}
d \\
1
\end{array}\right) T_{n}^{d-1} & \ldots & 1
\end{array}\right)
$$

In [5] it is shown that

$$
\mathcal{M}_{n}^{-1}=\left(\begin{array}{cccc}
1 & 0 & \ldots & 0 \\
T_{n} & 1 & \ldots & 0 \\
T_{n}^{2} & 2 T_{n} & \ldots & 0 \\
\ldots \ldots \ldots \ldots \ldots & \ldots & \\
T_{n}^{d} & \left(\begin{array}{l}
d \\
1
\end{array}\right) T_{n}^{d-1} & \ldots & 1
\end{array}\right)
$$

Note that $D_{n}^{d} \mathcal{M}_{n}^{-1}$ has entries in $K[x]$. Multiplying (6) on the left by $\mathcal{M}_{n}^{-1}$, we get

(7) $Q_{n}\left(x^{r^{n}}\right)\left(\begin{array}{c}1 \\ S \\ \vdots \\ S^{d}\end{array}\right)-\mathcal{M}_{n}^{-1}\left(\begin{array}{c}P_{n 0}\left(x^{r^{n}}\right) \\ P_{n 1}\left(x^{r^{n}}\right) \\ \vdots \\ P_{n d}\left(x^{r^{n}}\right)\end{array}\right)=x^{(d e+e+1) r^{n}} \mathcal{M}_{n}^{-1}\left(\begin{array}{c}0 \\ G_{n 1}\left(x^{r^{n}}\right) \\ \vdots \\ G_{n d}\left(x^{r^{n}}\right)\end{array}\right)$.

Multiplying (7) on the left by the row matrix $D_{n}^{d}\left(A_{0}, \ldots, A_{d}\right)$ we obtain

$$
U_{n}\left(\sum_{h=0}^{d} A_{h} S^{h}\right)-V_{n}=x^{(d e+e+1) r^{n}} H_{n}
$$

where

$$
\begin{aligned}
& U_{n}=D_{n}^{d} Q_{n}\left(x^{r^{n}}\right) \in K[x], \\
& V_{n}=\left(A_{0}, \ldots, A_{d}\right) D_{n}^{d} \mathcal{M}_{n}^{-1}\left(\begin{array}{c}
P_{n 0}\left(x^{r^{n}}\right) \\
P_{n 1}\left(x^{r^{n}}\right) \\
\vdots \\
P_{n d}\left(x^{r^{n}}\right)
\end{array}\right) \in K[x],
\end{aligned}
$$




$$
H_{n}=\left(A_{0}, \ldots, A_{d}\right) D_{n}^{d} \mathcal{M}_{n}^{-1}\left(\begin{array}{c}
0 \\
G_{n 1}\left(x^{r^{n}}\right) \\
\vdots \\
G_{n d}\left(x^{r^{n}}\right)
\end{array}\right) \in K[[x]] .
$$

Let $n$ be the positive integer such that

$$
r^{n-1} \leq c_{d-1} M<r^{n}
$$

Then, as $e=d L$ and $c_{d-1} \geq 1$,

$$
\operatorname{deg} V_{n} \leq M+d L r^{n}+d e r^{n}<(d e+e+1) r^{n} \text {. }
$$

Let $m$ be the least integer such that $\left(0, g_{n 1 m}, \ldots, g_{n d m}\right) \neq \mathbf{0}$. By Lemma 1, $m \leq \gamma$. Let

$$
\left(\begin{array}{c}
0 \\
g_{n 1 m} \\
\vdots \\
g_{n d m}
\end{array}\right)=\left(\begin{array}{c}
0 \\
\vdots \\
0 \\
g_{n i m} \\
\vdots \\
g_{n d m}
\end{array}\right), \quad g_{n i m} \neq 0
$$

Then, modulo $x^{(m+1) r^{n}}$, we have

$$
\begin{aligned}
& H_{n} \equiv D_{n}^{d}\left(A_{0}, \ldots, A_{d}\right) \mathcal{M}_{n}^{-1}\left(\begin{array}{c}
0 \\
\vdots \\
0 \\
g_{n i m} x^{m r^{n}} \\
\vdots \\
g_{n d m} x^{m r^{n}}
\end{array}\right)
\end{aligned}
$$

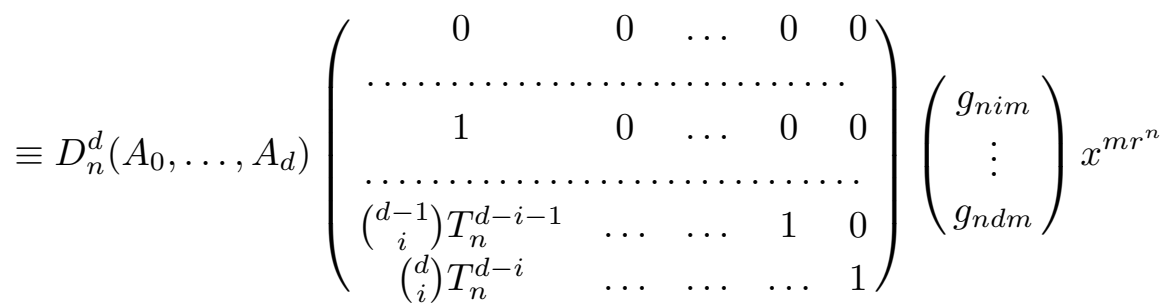

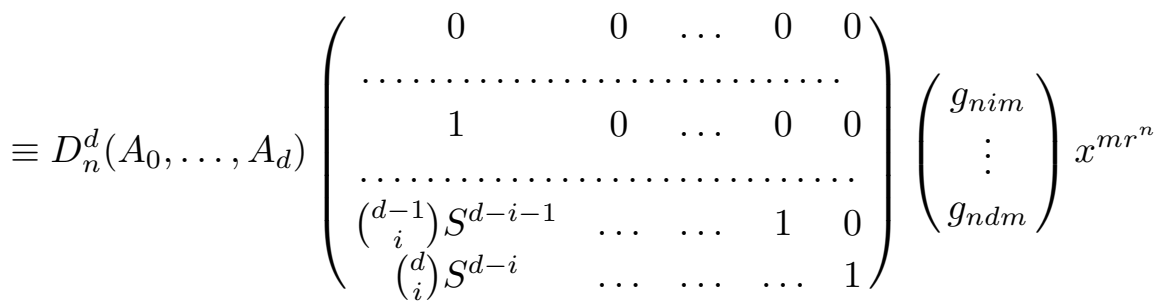

$$
\begin{aligned}
& \equiv D_{n}^{d}\left(B_{0}+B_{1} S+\ldots+B_{d-i} S^{d-i}\right) x^{m r^{n}} \text {, }
\end{aligned}
$$


where $B_{0}, \ldots, B_{d-i} \in K[x]$ and

$$
B_{d-i}=A_{d}\left(\begin{array}{c}
d \\
i
\end{array}\right) g_{n i m} \neq 0, \quad \operatorname{deg} B_{h} \leq M, \quad 0 \leq h \leq d-i .
$$

Since ord $D_{n}=0$, by (3) and (9) we obtain

$$
\operatorname{ord}\left(D_{n}^{d}\left(B_{0}+B_{1} S+\ldots+B_{d-i} S^{d-i}\right) x^{m r^{n}}\right) \leq c_{d-1} M+m r^{n}<(1+m) r^{n} .
$$

Hence $H_{n} \not \equiv 0 \bmod x^{(m+1) r^{n}}$. Suppose that $V_{n} \neq 0$. By (10) we get

$$
\text { ord } V_{n}<(d e+e+1) r^{n} \text {. }
$$

Therefore by (8), (9) we obtain

$$
\operatorname{ord}\left(\sum_{h=0}^{d} A_{h} S^{h}\right)<(d e+e+1) r^{n} \leq(d e+e+1) r c_{d-1} M .
$$

If $V_{n}=0$, by (8), (9) we obtain

$$
\operatorname{ord}\left(\sum_{h=0}^{d} A_{h} S^{h}\right)<(d e+e+1) r^{n}+(m+1) r^{n} \leq(d e+e+2+\gamma) r c_{d-1} M \text {. }
$$

Letting $c_{d}=(d e+e+2+\gamma) r c_{d-1}$, we obtain (2).

TheOREM 3. In addition to the hypotheses of Section 1, assume (1). Then $\Phi_{0}(\alpha)$ is transcendental.

Proof. We apply Theorem 1. Since

$$
\Phi_{n}\left(\alpha^{r^{n}}\right)=\Phi_{0}(\alpha)-\sum_{k=0}^{n-1} \frac{E_{k}\left(\alpha^{r^{k}}\right)}{F_{k}\left(\alpha^{r^{k}}\right)}
$$

property (I) is satisfied. We prove property (III). Suppose that $\operatorname{deg}_{x} P_{j}(x, s)$ $\leq N$ and ord $E\left(x, \sigma^{(n)}\right)=I_{n}$. Then

$$
I_{n} r^{n}=\operatorname{ord} E\left(x^{r^{n}}, \sigma^{(n)}\right)=\operatorname{ord}\left(\sum_{j=0}^{d} P_{j}\left(x^{r^{n}}, \sigma^{(n)}\right) \Phi_{n}\left(x^{r^{n}}\right)^{j}\right) .
$$

On the other hand,

$$
D_{n}^{d} \sum_{j=0}^{d} P_{j}\left(x^{r^{n}}, \sigma^{(n)}\right) \Phi_{n}\left(x^{r^{n}}\right)^{j}=\sum_{j=0}^{d} P_{j}\left(x^{r^{n}}, \sigma^{(n)}\right) D_{n}^{d}\left(S-T_{n}\right)^{j} .
$$

If $P_{0}\left(x, \sigma^{(n)}\right), \ldots, P_{d}\left(x, \sigma^{(n)}\right)$ are not all zero, by Theorem 2 we get

$$
\operatorname{ord} E\left(x^{r^{n}}, \sigma^{(n)}\right) \leq c_{d}\left(N r^{n}+d L r^{n}\right) .
$$

Therefore $I_{n} \leq c_{d}(N+d L)$, which proves (III). Property (II) results from the following. 
Lemma 2. For any $\theta>1$, there exists a positive integer $n_{0}$ such that

$$
\left\|\sigma_{l}^{(n)}\right\| \leq \theta^{l r^{n}} \quad \text { for any } n \geq n_{0} \text { and } l \geq 0 .
$$

Proof. Let $\sum_{k=0}^{\infty} a_{k} x^{k} \ll \sum_{k=0}^{\infty} b_{k} x^{k}$ mean $\left|a_{k}\right| \leq b_{k}$ for all $k$. Let $\theta>1$ and $k$ be greater than some constant depending on $\theta$ which will be determined below. We have

$$
\left\|a_{k l}\right\| \leq \theta^{r^{k}}, \quad\left\|b_{k l}\right\| \leq \theta^{r^{k}}, \quad 1 \leq l \leq L .
$$

Then

$$
\begin{aligned}
& E_{k}(x) \ll \theta^{r^{k}}\left(x+x^{2}+\ldots+x^{L}\right), \\
& \frac{1}{F_{k}(x)} \ll 1+\theta^{r^{k}}\left(x+\ldots+x^{L}\right)+\theta^{2 r^{k}}\left(x+\ldots+x^{L}\right)^{2}+\ldots
\end{aligned}
$$

Since $\left(x+\ldots+x^{L}\right)^{l} \ll L^{l}\left(x^{l}+x^{l+1}+\ldots\right)$, we get

$$
\begin{aligned}
\frac{E_{k}(x)}{F_{k}(x)} & \ll \theta^{r^{k}} L\left(x+x^{2}+\ldots\right)+\theta^{2 r^{k}} L^{2}\left(x^{2}+x^{3}+\ldots\right)+\ldots \\
& \ll \theta^{r^{k}} L x+\ldots+L^{l}\left(\theta^{r^{k}}+\ldots+\theta^{l r^{k}}\right) x^{l}+\ldots \\
& \ll\left(\theta^{2}\right)^{r^{k}} x+\ldots+\left(\theta^{2}\right)^{l r^{k}} x^{l}+\ldots
\end{aligned}
$$

So we obtain

$$
\begin{aligned}
\Phi_{n}(x) & \ll\left(\theta^{2}\right)^{r^{n}} x+\ldots+\left(\left[\log _{r} l\right]+1\right)\left(\theta^{2}\right)^{l r^{n}} x^{l}+\ldots \\
& \ll\left(\theta^{3}\right)^{r^{n}} x+\ldots+\left(\theta^{3}\right)^{l r^{n}} x^{l}+\ldots,
\end{aligned}
$$

if $n$ is sufficiently large. Hence $\left|\sigma_{l}^{(n)}\right| \leq\left(\theta^{3}\right)^{l r^{n}}$ for any $n \geq n_{0}$ and $l \geq 0$. In the same way, we have $\overline{\sigma_{l}^{(n)}} \leq\left(\theta^{3}\right)^{l r^{n}}$ for any $n \geq n_{0}$ and $l \geq 0$.

Since $\prod_{l=1}^{L} \operatorname{den}\left(a_{k l}\right) \leq \theta^{r^{k}}$ and $b_{k l} \in O_{K}, 1 \leq l \leq L$, we have

$$
\operatorname{den}\left(\sigma_{l}^{(n)}\right) \leq \theta^{r^{n}} \theta^{r^{n+1}} \ldots \theta^{r^{n+\left[\log _{r} l\right]}} \leq \theta^{r^{n+\left[\log _{r} l\right]}\left(1+r^{-1}+r^{-2}+\ldots\right)} \leq\left(\theta^{2}\right)^{l r^{n}}
$$

for any $n \geq n_{0}$ and $l \geq 0$.

Lemma 2 is proved and the proof of Theorem 3 is complete. To end this section, we prove Lemma 3, which will be used later.

LEMMA 3. Let

$$
f_{n}(x)=-\frac{B_{n}(x)}{A_{n}(x)}+\Phi_{n}(x),
$$

where $A_{n}(x), B_{n}(x) \in K[x], \operatorname{deg} A_{n}, \operatorname{deg} B_{n} \leq L, A_{n}(0)=1, B_{n}(0)=0$ and the $\log \|\|$ of the coefficients of $A_{n}(x), B_{n}(x)$ are $o\left(r^{n}\right)$. Let $I$ be a positive integer, and $\alpha \in K$ with $0<|\alpha|<1$. Then there exist positive numbers $\eta<1$ and $n_{0}$ such that

$$
0<\left|f_{n}\left(\alpha^{r^{n}}\right)\right|<\eta^{r^{n}} \text { ord } f_{n}(x)
$$

for every $n \geq n_{0}$ satisfying ord $f_{n}(x) \leq I$. 
Proof. Let $\theta>1$ and $B_{n}(x) / A_{n}(x)=\sum_{l=1}^{\infty} \tau_{l}^{(n)} x^{l}$. As in Lemma 2, we obtain $\left\|\tau_{l}^{(n)}\right\| \leq\left(\theta^{2}\right)^{l r^{n}}$. We put

$$
f_{n}(x)=\sum_{l=1}^{\infty}\left(-\tau_{l}^{(n)}+\sigma_{l}^{(n)}\right) x^{l}=a_{H} x^{H}+a_{H+1} x^{H+1}+\ldots, \quad a_{H} \neq 0 .
$$

Then $1 \leq H \leq I$ and by Lemma $2,\left\|a_{l}\right\| \leq\left(\theta^{4}\right)^{l r^{n}}$. We have

$$
f_{n}\left(\alpha^{r^{n}}\right)=a_{H} \alpha^{H r^{n}}\left(1+\frac{a_{H+1}}{a_{H}} \alpha^{r^{n}}+\frac{a_{H+2}}{a_{H}} \alpha^{2 r^{n}}+\ldots\right) .
$$

Since

$$
\left|\frac{a_{H+l}}{a_{H}}\right| \leq\left(\theta^{8[K: \mathbb{Q}]}\right)^{H r^{n}}\left(\theta^{4}\right)^{(H+l) r^{n}} \leq \theta^{(8[K: \mathbb{Q}]+4) H r^{n}} \theta^{4 l r^{n}},
$$

we obtain

$$
\left|\frac{a_{H+l}}{a_{H}} \alpha^{l r^{n}}\right| \leq\left(\theta^{8[K: \mathbb{Q}]+4}\right)^{I r^{n}}\left|\theta^{4} \alpha\right|^{l r^{n}} .
$$

We can choose $\theta>1$ such that

$$
\eta=\theta^{(8[K: \mathbb{Q}]+4) I}\left|\theta^{4} \alpha\right|<1 .
$$

Then

$$
\left|\frac{a_{H+l}}{a_{H}} \alpha^{l r^{n}}\right| \leq \eta^{l r^{n}}
$$

and so if $n$ is sufficiently large, then $0<\left|f_{n}\left(\alpha^{r^{n}}\right)\right|<2\left|\theta^{4} \alpha\right|^{H r^{n}}<\eta^{H r^{n}}$.

3. Proof of the Transcendence Criterion. We first prove a generalization of [3, Theorem 9.7, p. 113]. Let $\mathbf{K}$ be any commutative field with a nonarchimedean absolute value $\mid$, thus satisfying

$$
\begin{aligned}
& |x|=0 \Leftrightarrow x=0, \\
& |x y|=|x||y|, \\
& |x+y| \leq \max \{|x|,|y|\} .
\end{aligned}
$$

We suppose moreover that there exists in $\mathbf{K}$ a subring $\mathbf{A}$ with the following property:

$$
\text { for any } x \in \mathbf{A} \backslash\{0\}, \quad|x| \geq 1 \text {. }
$$

TheOrem 4. Let $\mathbf{K}$ be as above and $\alpha \in \mathbf{K}$. Suppose there exist $a, b, k, l$ $\in(0, \infty), h \geq 1$, an increasing sequence $\{g(n)\}_{n \geq 0}$ in $(0, \infty)$, and a sequence $\left\{\left(p_{n}, q_{n}\right)\right\}_{n \geq 0}$ in $\mathbf{A}^{2}$ such that

$$
\begin{array}{ll}
q_{n} p_{n+1}-q_{n+1} p_{n} \neq 0 & \text { for every } n \geq 0, \\
\left|q_{n}\right| \leq k g(n)^{a} & \text { for every } n \geq 0, \\
\left|q_{n} \alpha-p_{n}\right| \leq l / g(n) & \text { for every } n \geq 0,
\end{array}
$$




$$
\begin{aligned}
& \lim _{n \rightarrow \infty} g(n)=\infty, \\
& g(n+1) \leq b g(n)^{h} \quad \text { for every } n \geq 0 .
\end{aligned}
$$

Then for every $(p, q) \in \mathbf{A}^{2}$ with $q \neq 0$, we have

$$
|q \alpha-p| \geq c /|q|^{\mu}
$$

with $c=\left(k b^{a(h+1)} M^{a h^{2}}\right)^{-1}, \mu=a h^{2}$, and $M=\max \{l, g(0)\}$.

Proof. Let $(p, q) \in \mathbf{A}^{2}$, with $q \neq 0$, fixed. Let

$$
M=\max \{l, g(0)\} .
$$

Let $\nu$ be the least integer satisfying $|q| M / g(\nu)<1$. Such a $\nu$ exists because of (18). Moreover, as $|q| \geq 1$ because $q \in \mathbf{A} \backslash\{0\}$, we have $|q| M / g(0) \geq 1$, therefore $\nu \geq 1$. Thus $|q| M / g(\nu-1) \geq 1$, which implies $g(\nu-1) \leq|q| M$. By using (19), we obtain $g(\nu) \leq b(|q| M)^{h}$, and by using (19) again,

$$
g(\nu+1) \leq b^{h+1}(|q| M)^{h^{2}} .
$$

Now consider the determinant $\Delta_{\nu}=\left|\begin{array}{cc}q_{\nu} & p_{\nu} \\ q_{\nu+1} & p_{\nu+1}\end{array}\right|$. By (15), $\Delta_{\nu} \neq 0$, which means that the vectors $\left(q_{\nu}, p_{\nu}\right)$ and $\left(q_{\nu+1}, p_{\nu+1}\right)$ form a basis of $\mathbf{K}^{2}$. Hence one of the two determinants $\left|\begin{array}{ccc}q_{\nu} & p_{\nu} \\ q & p\end{array}\right|$ and $\left|\begin{array}{ccc}q_{\nu+1} & p_{\nu+1} \\ q & p\end{array}\right|$ is distinct from 0. Set $m=\nu$ or $m=\nu+1$, such that

$$
\delta_{m}=\left|\begin{array}{cc}
q_{m} & p_{m} \\
q & p
\end{array}\right| \neq 0 .
$$

As $\delta_{m} \in \mathbf{A} \backslash\{0\}$, we have by (14) $\left|p q_{m}-q p_{m}\right| \geq 1$. This means that $\left|q\left(q_{m} \alpha-p_{m}\right)-q_{m}(q \alpha-p)\right| \geq 1$. By using (13), we obtain

$$
\max \left\{\left|q\left(q_{m} \alpha-p_{m}\right)\right|,\left|q_{m}(q \alpha-p)\right|\right\} \geq 1 .
$$

But $\left|q\left(q_{m} \alpha-p_{m}\right)\right| \leq|q| l / g(m)$ by (17). As $l \leq M$ and $g$ is increasing, we have $\left|q\left(q_{m} \alpha-p_{m}\right)\right| \leq|q| M / g(\nu)<1$ by definition of $\nu$. Therefore in (23) the greatest number on the left-hand side cannot be $\left|q\left(q_{m} \alpha-p_{m}\right)\right|$, and (23) becomes

$$
\left|q_{m}\right||q \alpha-p| \geq 1 \Leftrightarrow|q \alpha-p| \geq 1 /\left|q_{m}\right| .
$$

By (16) we can write $\left|q_{m}\right| \leq k g(m)^{a} \leq k g(\nu+1)^{a}$. By using (21) we obtain

$$
\left|q_{m}\right| \leq k b^{a(h+1)}(|q| M)^{a h^{2}} .
$$

Therefore (24) becomes

$$
|q \alpha-p| \geq \frac{1}{k b^{a(h+1)} M^{a h^{2}}} \frac{1}{|q|^{a h^{2}}},
$$

which proves the theorem.

Now we specialize Theorem 4 to the following situation. Let $K$ be any commutative field, let $K[[x]]$ be the ring of formal power series with coef- 
ficients in $K$, and let $K((x))$ be the field of fractions of $K[[x]]$. It is well known that $K((x))$ is also the field of Laurent series with coefficients in $K$, which means that any $f \in K((x))^{\times}$can be written, in a unique form, as

$$
f(x)=\sum_{n \geq m} a_{n} x^{n} \quad \text { with } a_{m} \neq 0 .
$$

The valuation of $f \in K((x))^{\times}$is defined, as usual, by

$$
v(f)=m \text {. }
$$

It has the following properties:

$$
\begin{gathered}
v(f g)=v(f)+v(g), \\
v(f+g) \geq \min \{v(f), v(g)\} .
\end{gathered}
$$

Now fix any $\theta>1$. We define an absolute value on $K((x))$ by putting

$$
|f|=\theta^{-v(f)} \quad \text { if } f \neq 0, \quad|0|=0 .
$$

It is easily checked (and well known) that || satisfies (11)-(13).

Theorem 5. Let $K$ be a commutative field and $A, B, C \in \mathbb{R}, 0<A<B$, $C \geq 1$. Let $r \geq 2$ be an integer. Let $\{m(n)\}_{n \geq 0}$ be an increasing sequence of nonnegative integers satisfying $m(n+1)-m(n) \leq C$. Let $f \in K[[x]]$. Suppose that there exists a sequence $\left\{\left(P_{n}, Q_{n}\right)\right\}_{n \geq 0}$ in $K[x]^{2}$ satisfying

$$
\begin{array}{cc}
P_{n} Q_{n+1}-P_{n+1} Q_{n} \neq 0 & \text { for every } n \geq 0, \\
\operatorname{deg} Q_{n}, \operatorname{deg} P_{n} \leq A r^{m(n)} & \text { for every } n \geq 0, \\
v\left(Q_{n} f-P_{n}\right) \geq B r^{m(n)} & \text { for every } n \geq 0 .
\end{array}
$$

Then, for every $(P, Q) \in K[x]^{2}$ with $Q \neq 0$ and $\operatorname{deg} P, \operatorname{deg} Q \leq d, d \geq 1$,

$$
v(Q f-P) \leq\left(A r^{m(0)+2 C}\left(1+\frac{1}{B-A}\right)+1\right) d .
$$

Proof. We apply Theorem 4 with $\mathbf{K}$ replaced by $K((x))$ and

$$
\mathbf{A}=\left\{f \in K((x)) \mid f(x)=P\left(x^{-1}\right) \text { for some } P \in K[x]\right\} .
$$

For every $n \in \mathbb{N}$, put

$$
Q_{n}(x)=x^{\left[A r^{m(n)}\right]} \widetilde{Q}_{n}(x), \quad P_{n}(x)=x^{\left[A r^{m(n)}\right]} \widetilde{P}_{n}(x) .
$$

Then $\widetilde{P}_{n}, \widetilde{Q}_{n} \in \mathbf{A}$ for every $n \in \mathbb{N}$ and by (33),

$$
v\left(\widetilde{Q}_{n} f-\widetilde{P}_{n}\right) \geq(B-A) r^{m(n)} .
$$

By using the absolute value (30), we deduce from (31), (32) and (35) that

$$
\begin{array}{ll}
\widetilde{P}_{n} \widetilde{Q}_{n+1}-\widetilde{P}_{n+1} \widetilde{Q}_{n} \neq 0 & \text { for every } n \geq 0, \\
\left|\widetilde{Q}_{n}\right| \leq \theta^{A r^{m(n)}} & \text { for every } n \geq 0, \\
\left|\widetilde{Q}_{n} f-\widetilde{P}_{n}\right| \leq 1 / \theta^{(B-A) r^{m(n)}} & \text { for every } n \geq 0 .
\end{array}
$$


Therefore we can apply Theorem 4 with $g(n)=\theta^{(B-A) r^{m(n)}}, k=1, a=$ $A /(B-A), l=1, b=1, h=r^{C}, M=g(0)=\theta^{(B-A) r^{m(0)}}$. For every $(P, Q) \in K[x]^{2}$ with $\operatorname{deg} P, \operatorname{deg} Q \leq d, Q \neq 0$, put $P(x)=x^{d} \widetilde{P}(x)$ and $Q(x)=x^{d} \widetilde{Q}(x)$. Then $\widetilde{P}, \widetilde{Q} \in \mathbf{A}$ and by Theorem 4 we have

$$
|\widetilde{Q} f-\widetilde{P}| \geq \frac{1}{\theta^{A r^{m(0)+2 C}}} \frac{1}{|\widetilde{Q}|^{\frac{A}{B-A} r^{m(0)+2 C}}} .
$$

By taking logarithms, we get

$$
v(\widetilde{Q} f-\widetilde{P}) \leq A r^{m(0)+2 C}-\frac{A}{B-A} r^{m(0)+2 C} v(\widetilde{Q}) .
$$

But $v(\widetilde{Q}) \geq-d$, therefore

$$
v(\widetilde{Q} f-\widetilde{P}) \leq A r^{m(0)+2 C}\left(1+\frac{d}{B-A}\right)
$$

and finally

$v(Q f-P) \leq A r^{m(0)+2 C}\left(1+\frac{d}{B-A}\right)+d \leq\left(A r^{m(0)+2 C}\left(1+\frac{1}{B-A}\right)+1\right) d$,

because $d \geq 1$.

Now we are ready to prove the Transcendence Criterion. Let

$$
\Phi_{0}(x)=\sum_{k=0}^{\infty} \frac{E_{k}\left(x^{r^{k}}\right)}{F_{k}\left(x^{r^{k}}\right)}
$$

satisfy the assumptions in Section 1 and

$$
\Phi_{n}(x)=\sum_{k=0}^{\infty} \frac{E_{n+k}\left(x^{r^{k}}\right)}{F_{n+k}\left(x^{r^{k}}\right)} .
$$

It is clear that

$$
\Phi_{n}\left(x^{r}\right)=\Phi_{n-1}(x)-\frac{E_{n-1}(x)}{F_{n-1}(x)} .
$$

An easy induction shows that

$$
\Phi_{n}\left(x^{r^{n}}\right)=\Phi_{0}(x)-\sum_{k=0}^{n-1} \frac{E_{k}\left(x^{r^{k}}\right)}{F_{k}\left(x^{r^{k}}\right)} .
$$

We want to construct a sequence $\left\{\left(P_{n}, Q_{n}\right)\right\}_{n \geq 0}$ satisfying the hypotheses of Theorem 5. Consider the $(L, L)$ Padé approximants to $\Phi_{n}(x)$, that is, polynomials $A_{n}$ and $B_{n}$ satisfying $\operatorname{deg} A_{n}, \operatorname{deg} B_{n} \leq L$, and

$$
A_{n}(x) \Phi_{n}(x)-B_{n}(x)=O\left(x^{2 L+1}\right) \text {. }
$$


By using the so-called Siegel lemma (cf. [12, Lemma 1.4.2]), we may assume that the $\log \|\|$ of the coefficients of $A_{n}(x)$ and $B_{n}(x)$ are $o\left(r^{n}\right)$. Define

$$
D_{n}(x)=\left|\begin{array}{cc}
A_{n}(x) & B_{n}(x) \\
A_{n+1}\left(x^{r}\right) & A_{n+1}\left(x^{r}\right) \frac{E_{n}(x)}{F_{n}(x)}+B_{n+1}\left(x^{r}\right)
\end{array}\right| .
$$

Lemma 4. Suppose that $D_{n}(x) \neq 0$. Then

$$
\operatorname{ord}\left(A_{n}(x) \Phi_{n}(x)-B_{n}(x)\right) \leq r(2 L+1) .
$$

Proof. Suppose that

$$
A_{n}(x) \Phi_{n}(x)-B_{n}(x)=O\left(x^{q}\right)
$$

with $q>r(2 L+1)$. We also have, by $(42)$,

$$
A_{n+1}(x) \Phi_{n+1}(x)-B_{n+1}(x)=O\left(x^{2 L+1}\right) .
$$

Replacing $x$ by $x^{r}$ and using (40), we obtain

$$
A_{n+1}\left(x^{r}\right) \Phi_{n}(x)-\left(A_{n+1}\left(x^{r}\right) \frac{E_{n}(x)}{F_{n}(x)}+B_{n+1}\left(x^{r}\right)\right)=O\left(x^{r(2 L+1)}\right) .
$$

Multiply the first column of $D_{n}(x)$ by $\Phi_{n}(x)$ and subtract it from the second one. By (44) and (45), we see that $D_{n}(x)=O\left(x^{r(2 L+1)}\right)$. This means that

$$
\begin{aligned}
F_{n}(x) D_{n}(x) & =\left|\begin{array}{cc}
A_{n}(x) & B_{n}(x) \\
A_{n+1}\left(x^{r}\right) F_{n}(x) & A_{n+1}\left(x^{r}\right) E_{n}(x)+B_{n+1}\left(x^{r}\right) F_{n}(x)
\end{array}\right| \\
& =O\left(x^{r(2 L+1)}\right) .
\end{aligned}
$$

But this determinant is a polynomial of degree at most $L(r+2)$. As $L(r+2)<$ $r(2 L+1)$, we have $D_{n}(x)=0$. This contradiction proves Lemma 4 .

Now we construct the sequence $\left\{\left(P_{n}, Q_{n}\right)\right\}_{n \geq 0}$. If we replace $x$ by $x^{r^{n}}$ in (42) and use the functional equation (41) we obtain

$$
Q_{n}^{*}(x) \Phi_{0}(x)-P_{n}^{*}(x)=O\left(x^{(2 L+1) r^{n}}\right),
$$

where

$$
\begin{aligned}
& Q_{n}^{*}(x)=A_{n}\left(x^{r^{n}}\right) \prod_{k=0}^{n-1} F_{k}\left(x^{r^{k}}\right), \\
& P_{n}^{*}(x)=\left(A_{n}\left(x^{r^{n}}\right) \sum_{k=0}^{n-1} \frac{E_{k}\left(x^{r^{k}}\right)}{F_{k}\left(x^{r^{k}}\right)}+B_{n}\left(x^{r^{n}}\right)\right) \prod_{k=0}^{n-1} F_{k}\left(x^{r^{k}}\right) .
\end{aligned}
$$

It is clear that

$$
\operatorname{deg} Q_{n}^{*} \leq \frac{r L}{r-1} r^{n}, \quad \operatorname{deg} P_{n}^{*} \leq \frac{r L}{r-1} r^{n} .
$$

As $r L /(r-1) \leq 2 L$ for every $r \geq 2$, we see that the sequence $\left\{\left(P_{n}, Q_{n}\right)\right\}_{n \geq 0}$ $=\left\{\left(P_{m(n)}^{*}, Q_{m(n)}^{*}\right)\right\}_{n \geq 0}$ satisfies hypotheses (32) and (33) of Theorem 5 for 
every increasing sequence $\{m(n)\}_{n \geq 0}$. It remains to study condition (31) in Theorem 5. We need the following lemma.

LEMMA 5. For every $n \geq 0$, put

$$
\Delta_{n}(x)=\left|\begin{array}{cc}
Q_{n}^{*}(x) & P_{n}^{*}(x) \\
Q_{n+1}^{*}(x) & P_{n+1}^{*}(x)
\end{array}\right| .
$$

Then $\Delta_{n}(x)=0$ if and only if $D_{n}(x)=0$, that is,

$$
\frac{E_{n}(x)}{F_{n}(x)}=\frac{B_{n}(x)}{A_{n}(x)}-\frac{B_{n+1}\left(x^{r}\right)}{A_{n+1}\left(x^{r}\right)} .
$$

Proof. We have

$$
\begin{aligned}
& \Delta_{n}(x)=0 \\
& \Leftrightarrow\left|\begin{array}{cc}
A_{n}\left(x^{r^{n}}\right) & A_{n}\left(x^{r^{n}}\right) \sum_{k=0}^{n-1} \frac{E_{k}\left(x^{r^{k}}\right)}{F_{k}\left(x^{r^{k}}\right)}+B_{n}\left(x^{r^{n}}\right) \\
A_{n+1}\left(x^{r^{n+1}}\right) & A_{n+1}\left(x^{r^{n+1}}\right) \sum_{k=0}^{n} \frac{E_{k}\left(x^{r^{k}}\right)}{F_{k}\left(x^{r^{k}}\right)}+B_{n+1}\left(x^{r^{n+1}}\right)
\end{array}\right|=0 \\
& \Leftrightarrow\left|\begin{array}{cc}
A_{n}\left(x^{r^{n}}\right) & B_{n}\left(x^{r^{n}}\right) \\
A_{n+1}\left(x^{r^{n+1}}\right) & A_{n+1}\left(x^{r^{n+1}}\right) \frac{E_{n}\left(x^{r^{n}}\right)}{F_{n}\left(x^{r^{n}}\right)}+B_{n+1}\left(x^{r^{n+1}}\right)
\end{array}\right|=0 \\
& \Leftrightarrow\left|\begin{array}{cc}
A_{n}(x) & B_{n}(x) \\
A_{n+1}\left(x^{r}\right) & A_{n+1}\left(x^{r}\right) \frac{E_{n}(x)}{F_{n}(x)}+B_{n+1}\left(x^{r}\right)
\end{array}\right|=0 \Leftrightarrow D_{n}(x)=0 \\
& \Leftrightarrow\left|\begin{array}{cc}
1 & \frac{B_{n}(x)}{A_{n}(x)} \\
1 & \frac{E_{n}(x)}{F_{n}(x)}+\frac{B_{n+1}\left(x^{r}\right)}{A_{n+1}\left(x^{r}\right)}
\end{array}\right|=0
\end{aligned}
$$

which is the desired conclusion.

The following theorem is a precise version of the Transcendence Criterion.

THEOREM 6. Under the hypotheses of Section $1, \Phi_{0}(\alpha)$ is algebraic if and only if $\Delta_{n}(x)=0$ for every $n \geq N$, that is,

$$
\frac{E_{n}(x)}{F_{n}(x)}=\frac{B_{n}(x)}{A_{n}(x)}-\frac{B_{n+1}\left(x^{r}\right)}{A_{n+1}\left(x^{r}\right)} \quad \text { for every } n \geq N \text {. }
$$

Proof. Suppose that there exist infinitely many $n$ satisfying $\Delta_{n}(x) \neq 0$. Denote by $\{m(n)\}_{n \geq 0}$ the sequence satisfying

$$
\Delta_{m(n)}(x) \neq 0, \quad \Delta_{k}(x)=0
$$


for every $n \geq 0$ and every $k$ with $m(n)<k<m(n+1)$. Then two cases can occur.

(I) $m(n+1)-m(n) \leq C$ for some constant $C>0$. Then it is clear that the determinant

$$
\delta_{n}=\left|\begin{array}{cc}
Q_{m(n)}^{*}(x) & P_{m(n)}^{*}(x) \\
Q_{m(n+1)}^{*}(x) & P_{m(n+1)}^{*}(x)
\end{array}\right|=\left|\begin{array}{cc}
Q_{n}(x) & P_{n}(x) \\
Q_{n+1}(x) & P_{n+1}(x)
\end{array}\right|
$$

is not zero. Therefore condition (31) in Theorem 5 is fulfilled, and we can apply Theorem 3 . Hence $\Phi_{0}(\alpha)$ is transcendental.

(II) $\lim \sup (m(n+1)-m(n))=\infty$. In this case, by using Lemma 5 we have

$$
\frac{E_{k}(x)}{F_{k}(x)}=\frac{B_{k}(x)}{A_{k}(x)}-\frac{B_{k+1}\left(x^{r}\right)}{A_{k+1}\left(x^{r}\right)}
$$

for every $k$ satisfying $m(n)<k<m(n+1)$, so that

$$
\sum_{k=m(n)+1}^{m(n+1)-1} \frac{E_{k}\left(x^{r^{k}}\right)}{F_{k}\left(x^{r^{k}}\right)}=\frac{B_{m(n)+1}\left(x^{r^{m(n)+1}}\right)}{A_{m(n)+1}\left(x^{r^{m(n)+1}}\right)}-\frac{B_{m(n+1)}\left(x^{r^{m(n+1)}}\right)}{A_{m(n+1)}\left(x^{r^{m(n+1)}}\right)} .
$$

Thus we have

$$
\begin{aligned}
\Phi_{0}(x)= & \sum_{k=0}^{m(n)} \frac{E_{k}\left(x^{r^{k}}\right)}{F_{k}\left(x^{r^{k}}\right)}+\frac{B_{m(n)+1}\left(x^{r^{m(n)+1}}\right)}{A_{m(n)+1}\left(x^{r^{m(n)+1}}\right)} \\
& -\frac{B_{m(n+1)}\left(x^{r^{m(n+1)}}\right)}{A_{m(n+1)}\left(x^{r^{m(n+1)}}\right)}+\Phi_{m(n+1)}\left(x^{r^{m(n+1)}}\right) .
\end{aligned}
$$

Let

$$
f_{m(n+1)}(x)=-\frac{B_{m(n+1)}(x)}{A_{m(n+1)}(x)}+\Phi_{m(n+1)}(x) .
$$

As $\Delta_{m(n+1)}(x) \neq 0$, we have $D_{m(n+1)}(x) \neq 0$ by Lemma 5 . Therefore by Lemma 4,

$$
\text { ord } f_{m(n+1)}(x) \leq \operatorname{ord}\left(A_{m(n+1)}(x) \Phi_{m(n+1)}(x)-B_{m(n+1)}(x)\right) \leq r(2 L+1) .
$$

Since ord $\Phi_{n}(x) \geq 1$, we may assume that $A_{m(n+1)}(0)=1$ and $B_{m(n+1)}(0)$ $=0$. Applying Lemma 3, we see that, for every sufficiently large $n$,

$$
0<\left|f_{m(n+1)}\left(\alpha^{r^{m(n+1)}}\right)\right|<\eta^{(2 L+1) r^{m(n+1)}} .
$$

By (46) we have

$$
f_{m(n+1)}\left(\alpha^{r^{m(n+1)}}\right)=\Phi_{0}(\alpha)-\sum_{k=0}^{m(n)} \frac{E_{k}\left(\alpha^{r^{k}}\right)}{F_{k}\left(\alpha^{r^{k}}\right)}-\frac{B_{m(n)+1}\left(\alpha^{r^{m(n)+1}}\right)}{A_{m(n)+1}\left(\alpha^{r^{m(n)+1}}\right)} .
$$


If $\Phi_{0}(\alpha)$ is algebraic, then $f_{m(n+1)}\left(\alpha^{r^{m(n+1)}}\right)$ is also algebraic and we can see easily

$$
\left\|f_{m(n+1)}\left(\alpha^{r^{m(n+1)}}\right)\right\| \leq C^{r^{m(n)}}
$$

where $C>1$ is some constant. The inequalities (47), (48) contradict the fundamental inequalities recalled in Section 1. Hence we proved that $\Phi_{0}(\alpha)$ is transcendental in both cases. The converse is trivial.

4. Technical lemmas. In order to apply Theorem 6 , we will need to get some knowledge about polynomials $A_{n}$ and $B_{n}$ satisfying $\left(A_{n}, B_{n}\right)=1$ and

$$
\frac{E_{n}(x)}{F_{n}(x)}=\frac{B_{n}(x)}{A_{n}(x)}-\frac{B_{n+1}\left(x^{r}\right)}{A_{n+1}\left(x^{r}\right)}=\frac{B_{n}(x) A_{n+1}\left(x^{r}\right)-A_{n}(x) B_{n+1}\left(x^{r}\right)}{A_{n}(x) A_{n+1}\left(x^{r}\right)}
$$

with $\operatorname{deg} E_{n}, \operatorname{deg} F_{n}, \operatorname{deg} A_{n}, \operatorname{deg} B_{n} \leq L, A_{n}(0)=1, B_{n}(0)=0$. We will also assume that $L \geq r-1$, because we will see later (in Section 5.1) that the case $L<r-1$ is easy to handle. The main result in this section will be Lemma 10, which asserts that, under some additional assumptions, $A_{n}(x) \mid A_{n+1}\left(x^{r}\right)$.

LEMMA 6. If $B_{n+1}(x) \neq 0$ and (49) holds, then

$$
\operatorname{deg} A_{n+1}(x) \leq 2 L / r .
$$

Proof. Suppose that $\operatorname{deg} A_{n+1}(x)>2 L / r$. Then $\operatorname{deg} A_{n+1}\left(x^{r}\right) \geq 2 L+1$. As $\operatorname{deg} F_{n} \leq L$ in (49), there exists $q(x) \in K[x]$ with $\operatorname{deg} q(x) \geq L+1$ such that

$$
q(x) \mid A_{n+1}\left(x^{r}\right) \quad \text { and } \quad q(x) \mid B_{n}(x) A_{n+1}\left(x^{r}\right)-A_{n}(x) B_{n+1}\left(x^{r}\right) .
$$

As $B_{n+1}\left(x^{r}\right) \neq 0$ and $\left(A_{n+1}, B_{n+1}\right)=1$, from (50) we have $q(x) \mid A_{n}(x)$, a contradiction with $\operatorname{deg} A_{n} \leq L$.

LEMMA 7. Suppose there exist infinitely many $n$ such that $E_{n}(x) \neq 0$. If there exists $n \in \mathbb{N}$ such that $B_{n}(x)=0$, then there exists $(m, d) \in \mathbb{N}^{2}$ such that $\operatorname{deg} E_{m}=d r$ and $m \geq n$.

Proof. Suppose that $B_{n}(x)=0$. If $B_{n+1}(x)=0$, then by (49) we have $E_{n}(x)=0$. Therefore there exists $m \geq n$ such that $B_{m}(x)=0, B_{m+1}(x)$ $\neq 0$. Since $B_{m+1}(0)=0$ and $B_{m+1}(x) \neq 0, d=\operatorname{deg} B_{m+1}(x) \geq 1$ and by (49), we have

$$
\frac{E_{m}(x)}{F_{m}(x)}=-\frac{B_{m+1}\left(x^{r}\right)}{A_{m+1}\left(x^{r}\right)}
$$

which implies $\operatorname{deg} E_{m}(x)=d r$.

LEMMA 8. If $B_{n}(x) \neq 0$ and $\operatorname{deg} F_{n-1}>L-r$, then $\operatorname{deg} A_{n}(x) \geq 1$. 
Proof. Assume that $\operatorname{deg} A_{n}=0$, that is, $A_{n}(x)=1$. Then from (49),

$$
\frac{E_{n-1}(x)}{F_{n-1}(x)}=\frac{B_{n-1}(x)-B_{n}\left(x^{r}\right) A_{n-1}(x)}{A_{n-1}(x)} .
$$

As the right-hand side is irreducible, we have $A_{n-1}(x)=F_{n-1}(x)$ and

$$
E_{n-1}(x)=B_{n-1}(x)-B_{n}\left(x^{r}\right) A_{n-1}(x) .
$$

Hence $\operatorname{deg} B_{n}\left(x^{r}\right) A_{n-1}(x)>r+(L-r)=L$ and $\operatorname{deg} E_{n-1}>L$, a contradiction.

Lemma 9. Suppose that there exist infinitely many $n$ such that $E_{n}(x)$ $\neq 0$ and

$$
\begin{aligned}
& r \geq L / 2+1, \\
& \operatorname{deg} E_{n} \text { is not a multiple of } r \text { for every } n \geq N, \\
& \operatorname{deg} F_{n}>L-r \text { for every } n \geq N .
\end{aligned}
$$

Then for large $n, B_{n}(x) \neq 0$ and $h=\operatorname{deg} A_{n}$ is a constant satisfying

$$
1 \leq h \leq 2 L / r .
$$

Remark. We put deg $0=-1$.

Proof. Let $n \geq N+1$. Then $B_{n}(x) \neq 0$ by Lemma 7 . Suppose that $\operatorname{deg} A_{n+1}>\operatorname{deg} A_{n}$. Then (50) holds with $\operatorname{deg} q(x) \geq\left(\operatorname{deg} A_{n}+1\right) r-L$. As $q(x) \mid A_{n}(x)$, we have

$$
\operatorname{deg} A_{n} \geq \operatorname{deg} q \geq r \operatorname{deg} A_{n}+r-L,
$$

which implies $(r-1) \operatorname{deg} A_{n} \leq L-r$. As $\operatorname{deg} A_{n} \geq 1$ by (53) and Lemma 8, we obtain $r-1 \leq L-r$, that is, $r \leq(L+1) / 2$, a contradiction with (51). Hence $\operatorname{deg} A_{n+1} \leq \operatorname{deg} A_{n}$, and so $h=\operatorname{deg} A_{n}$ is a constant for large $n$. We have $1 \leq \operatorname{deg} A_{n} \leq 2 L / r$ by Lemma 6 .

LEMma 10. Under the assumptions of Lemma 9, we have $h=1$ or $A_{n}(x) \mid A_{n+1}\left(x^{r}\right)$ for every large $n$. Moreover, if $\operatorname{deg} A_{n}=\operatorname{deg} A_{n+1}=1$ and $A_{n}(x)$ does not divide $A_{n+1}\left(x^{r}\right)$, then $\operatorname{deg} F_{n}=r+1$.

Proof. Assume that $A_{n}(x)$ does not divide $A_{n+1}\left(x^{r}\right)$ for some large $n$. (50) holds with $\operatorname{deg} q \geq h r-L$. As before, we have $q(x) \mid A_{n}(x)$. Then $\operatorname{deg} q(x) \leq \operatorname{deg} A_{n}(x)=h$. If $\operatorname{deg} q(x)=h$, then $A_{n}(x) \mid A_{n+1}\left(x^{r}\right)$, a contradiction. So $\operatorname{deg} q(x)<h$ and $h>h r-L$. Hence $h<L /(r-1) \leq 2$ by (51). Therefore $h=1$.

If $\operatorname{deg} A_{n}=\operatorname{deg} A_{n+1}=1$ and $A_{n}(x)$ does not divide $A_{n+1}\left(x^{r}\right)$, then $A_{n}(x)$ and $A_{n+1}\left(x^{r}\right)$ are prime to each other, which implies $\operatorname{deg} F_{n}=r+1$ by (49).

We end this section by two lemmas giving polynomials $A_{n}$ satisfying $A_{n}(x) \mid A_{n+1}\left(x^{r}\right)$ in the cases $h=1$ and $h=2$. 
Lemma 11. Assume that $A_{n}(x) \mid A_{n+1}\left(x^{r}\right)$, and $\operatorname{deg} A_{n}=1$ for every $n \geq N$. Then, for every $n \geq N$,

$$
A_{n}(x)=1-a^{r^{n}} x \quad \text { for some } a \in \bar{K} .
$$

Proof. Put $A_{n}(x)=1-q_{n} x$. Then $A_{n+1}\left(q_{n}^{-r}\right)=0$, which implies that $1-q_{n+1} q_{n}^{-r}=0$, that is, $q_{n+1}=q_{n}^{r}$. Therefore there exists $a \in \bar{K}$ such that $q_{n}=a^{r^{n}}$.

Lemma 12. Assume that $A_{n}(x) \mid A_{n+1}\left(x^{r}\right)$, and $\operatorname{deg} A_{n}=2$ for every $n \geq N$. Then only two cases can occur:

(i) There exist $a, b \in \bar{K}$ such that for every $n \geq N$,

$$
A_{n}(x)=\left(1-a^{r^{n}} x\right)\left(1-b^{r^{n}} x\right) .
$$

(ii) There exist $M \geq N, a \in \bar{K}$ and a sequence $\left\{\omega_{n}\right\}_{n \geq 0}$ of $r$ th roots of unity such that for every $n \geq M$,

$$
A_{n}(x)=\left(1-a^{r^{n}} x\right)\left(1-\omega_{n} a^{r^{n}} x\right) .
$$

Proof. Put $A_{n}(x)=\left(1-q_{n} x\right)\left(1-q_{n}^{\prime} x\right)$. Then

$$
\left(1-q_{n} x\right)\left(1-q_{n}^{\prime} x\right) \mid\left(1-q_{n+1} x^{r}\right)\left(1-q_{n+1}^{\prime} x^{r}\right) .
$$

Then, as in the proof of Lemma 11, we may assume $q_{n+1}=q_{n}^{r}$ and $q_{n}=a^{r^{n}}$ for every $n \geq N$. We now have

$$
1-q_{n}^{\prime} x \mid\left(\sum_{k=0}^{r-1}\left(q_{n} x\right)^{k}\right)\left(1-q_{n+1}^{\prime} x^{r}\right) .
$$

Therefore we have $q_{n+1}^{\prime}=\left(q_{n}^{\prime}\right)^{r}$ or $q_{n}^{\prime}=\omega_{n} q_{n}$ with $\omega_{n}^{r}=1$. If there exists $M \geq N$ such that $q_{M}^{\prime}=\omega_{M} q_{M}$ with $\omega_{M}^{r}=1$, then $q_{M+1}^{\prime}=\left(q_{M}^{\prime}\right)^{r}=$ $\left(\omega_{M} q_{M}\right)^{r}=q_{M}^{r}=q_{M+1}$ or $q_{M+1}^{\prime}=\omega_{M+1} q_{M+1}$ with $\omega_{M+1}=1$. In both cases we see that $q_{M+1}^{\prime}=\omega_{M+1} q_{M+1}$ with $\omega_{M+1}^{r}=1$ and by induction (55) holds. If $q_{n+1}^{\prime}=\left(q_{n}^{\prime}\right)^{r}$ for every $n \geq N$, then (54) holds.

\section{Examples}

\subsection{The case $L<r-1$}

THEOREM 7. Let $\Phi_{0}(x)$ satisfy the assumptions of Section 1 . If $L<r-1$ and $E_{n}(x) \neq 0$ for infinitely many $n$, then $\Phi_{0}(x) \notin K(x)$. Therefore, for every algebraic $\alpha$ with $0<|\alpha|<1$ and $F_{k}\left(\alpha^{r^{k}}\right) \neq 0$ for every $k, \Phi_{0}(\alpha)$ is transcendental. Then

Proof. Suppose that $\Phi_{0}(x)=P(x) / Q(x)$, where $P(x), Q(x) \in K[x]$.

$$
\frac{P(x)}{Q(x)}-\sum_{k=0}^{n-1} \frac{E_{k}\left(x^{r^{k}}\right)}{F_{k}\left(x^{r^{k}}\right)}=\Phi_{n}\left(x^{r^{n}}\right) .
$$


If $E_{n}(x) \neq 0$, then $\Phi_{n}\left(x^{r^{n}}\right) \neq 0$ and ord $\Phi_{n}\left(x^{r^{n}}\right) \geq r^{n}$. On the other hand, $Q(x) \Phi_{n}\left(x^{r^{n}}\right) \prod_{k=0}^{n-1} F_{k}\left(x^{r^{k}}\right)$ is a polynomial of degree less than

$$
\operatorname{deg} P(x)+\operatorname{deg} Q(x)+\frac{L}{r-1} r^{n} .
$$

Therefore we have

$$
r^{n} \leq \operatorname{deg} P(x)+\operatorname{deg} Q(x)+\frac{L}{r-1} r^{n},
$$

which is a contradiction if $n$ is large.

5.2. The case $L=r-1$. Let

$$
F_{k}(x)=\sum_{i=0}^{r-1} x^{i}, \quad E_{k}(x)=r^{k} x F_{k}^{\prime}(x)=r^{k} \sum_{i=1}^{r-1} i x^{i} .
$$

Then

Hence

$$
\frac{E_{k}(x)}{F_{k}(x)}=\frac{r^{k}(1-x) \sum_{i=1}^{r-1} i x^{i}}{(1-x) \sum_{i=0}^{r-1} x^{i}}=r^{k}\left(\frac{x}{1-x}-\frac{r x^{r}}{1-x^{r}}\right) .
$$

$$
\Phi_{0}(x)=\sum_{k=0}^{\infty} \frac{E_{k}\left(x^{r^{k}}\right)}{F_{k}\left(x^{r^{k}}\right)}=\frac{x}{1-x} .
$$

Note that this formula can also be obtained from Corollary 4.1 in Duverney and Shiokawa [7], by taking $d=r, c=1, P(x)=1-x, Q(x)=\sum_{i=0}^{r-1} x^{i}$. Moreover for any $\omega \in K, \Phi_{0}(\omega x)$ is also in $K(x)$. The next theorem asserts that when $L=r-1$, only such functions are rational functions.

TheORem 8. Let $\Phi_{0}(x)$ satisfy the assumptions of Section 1. Suppose that $L=r-1, E_{n}(x) \neq 0$ for infinitely many $n$ and $\Phi_{0}(x) \in K(x)$. Then there exist a constant $c$, a root of unity $\omega$ and a positive integer $N$ such that

$$
\frac{E_{n}\left(x^{r^{n}}\right)}{F_{n}\left(x^{r^{n}}\right)}=c r^{n}\left(\frac{(\omega x)^{r^{n}}}{1-(\omega x)^{r^{n}}}-\frac{r(\omega x)^{r^{n+1}}}{1-(\omega x)^{r^{n+1}}}\right) \quad \text { for every } n \geq N .
$$

Proof. Since $\Phi_{0}(x) \in K(x), \Phi_{0}(\alpha)$ is algebraic, and from Theorem 6 , we have (49) for every $n \geq N$. Since $L=r-1$, Lemma 9 applies and $h=\operatorname{deg} A_{n}=1$ for every large $n$. By Lemmas 10 and 11, $A_{n}(x)=1-a^{r^{n}} x$. Therefore (49) can be written as

$$
\frac{E_{n}(x)}{F_{n}(x)}=\frac{B_{n}(x) \sum_{k=0}^{r-1} a^{k r^{n}} x^{k}-B_{n+1}\left(x^{r}\right)}{1-\left(a^{r n} x\right)^{r}} .
$$

Assume $\operatorname{deg} B_{n+1} \geq 2$. As $\operatorname{deg}\left(B_{n}(x) \sum_{k=0}^{r-1} a^{k r^{n}} x^{k}\right) \leq L+r-1=2 r-2$, the degree of the numerator is at least $2 r$. Therefore

$$
\operatorname{deg} F_{n}(x)\left(B_{n}(x) \sum_{k=0}^{r-1} a^{k r^{n}} x^{k}-B_{n+1}\left(x^{r}\right)\right) \geq 2 r,
$$


which is a contradiction because $\operatorname{deg} E_{n}(x)\left(1-\left(a^{r^{n}} x\right)^{r}\right) \leq L+r=2 r-1$. Hence $\operatorname{deg} B_{n+1} \leq 1$. As $B_{n}(0)=0$, we have $B_{n}(x)=b_{n} x$ for every large $n$, and (56) becomes

$$
\frac{E_{n}(x)}{F_{n}(x)}=\frac{b_{n} x \sum_{k=0}^{r-1} a^{k r^{n}} x^{k}-b_{n+1} x^{r}}{1-\left(a^{r^{n}} x\right)^{r}} .
$$

As $\operatorname{deg} F_{n} \leq L=r-1$, at least one linear divisor of $1-\left(a^{r^{n}} x\right)^{r}$ must divide both the numerator and the denominator; it is $1-a^{r^{n}} x=A_{n}(x)$. Hence the numerator must vanish for $x=a^{-r^{n}}$, whence $b_{n} r a^{-r^{n}}-b_{n+1} a^{-r^{n+1}}=0$, that is, $b_{n+1}=r a^{(r-1) r^{n}} b_{n}$. Therefore $b_{n}=c r^{n} a^{r^{n}}$, and $a$ must be a root of unity because of Kronecker's theorem and the growth condition on the coefficients of $E_{n}$ and $F_{n}$.

EXAMPLE 1. Let $r=2$ and $L=1$. Let $K$ be an algebraic number field and

$$
\Phi_{0}(x)=\sum_{k=0}^{\infty} \frac{x^{2^{k}}}{1+b_{k} x^{2^{k}}},
$$

where $b_{k} \in K$ is an integer for every $k$ and $\log \left\|b_{k}\right\|=o\left(2^{k}\right)$. By Theorem 8, $\Phi_{0}(x) \notin K(x)$. Hence $\Phi_{0}(\alpha)$ is transcendental for any $\alpha \in K, 0<|\alpha|<1$. By taking $b_{k} \in \mathbb{Z}$ and $\alpha=1 / a, a \in \mathbb{Z},|a|>1$, we obtain the special case dealt with in [5].

5.3. The case $L=r$. The case $L=r$ is much more complicated. First, the rational sums in Theorem 8 can be used to obtain many new series. For $r=L=2$ for example, we have

$$
\sum_{n=0}^{\infty} \frac{2^{n} x^{2^{n}}}{1+x^{2^{n}}}=\frac{x}{1-x},
$$

so that for every $\alpha_{1}, \alpha_{2} \in K$ and roots of unity $\omega_{1}, \omega_{2}$,

$$
\begin{aligned}
& \alpha_{1} \sum_{n=0}^{\infty} \frac{2^{n}\left(\omega_{1} x\right)^{2^{n}}}{1+\left(\omega_{1} x\right)^{2^{n}}}+\alpha_{2} \sum_{n=0}^{\infty} \frac{2^{n}\left(\omega_{2} x\right)^{2^{n}}}{1+\left(\omega_{2} x\right)^{2^{n}}} \\
&=\sum_{n=0}^{\infty} \frac{2^{n} x^{2^{n}}\left(\alpha_{1} \omega_{1}^{2^{n}}+\alpha_{2} \omega_{2}^{2^{n}}+\left(\omega_{1} \omega_{2}\right)^{2^{n}}\left(\alpha_{1}+\alpha_{2}\right) x^{2^{n}}\right)}{\left(1+\omega_{1}^{2^{n}} x^{2^{n}}\right)\left(1+\omega_{2}^{2^{n}} x^{2^{n}}\right)}
\end{aligned}
$$

is a rational function. Another type of weird series is the following. Let $\left\{a_{n}\right\}$ and $\left\{b_{n}\right\}$ be any sequences in $K$. Put

$$
\begin{aligned}
E_{2 n}(x) & =a_{n} x^{r}, & F_{2 n}(x) & =1+b_{n} x^{r}, \\
E_{2 n+1}(x) & =-a_{n} x, & F_{2 n+1}(x) & =1+b_{n} x .
\end{aligned}
$$

Then obviously $\sum_{n=0}^{\infty} E_{n}\left(x^{r^{n}}\right) / F_{n}\left(x^{r^{n}}\right)=0$.

In order to avoid these cases, we will assume that $1 \leq \operatorname{deg} E_{n}<r$. 
TheOREm 9. Let $\Phi_{0}(x)$ satisfy the assumptions of Section 1. Suppose that $L=r, \operatorname{deg} E_{n}<r, \operatorname{deg} F_{n}=r$ for every large $n, E_{n}(x) \neq 0$ for infinitely many $n$, and $\Phi_{0}(x) \in K(x)$. Then only three cases can occur:

(i) There exist a root of unity $\omega$ and a constant $c$ such that for every large $n$,

$$
E_{n}(x)=c \sum_{k=1}^{r-1}\left(\omega^{r^{n}} x\right)^{k}, \quad F_{n}(x)=1-\left(\omega^{r^{n}} x\right)^{r} .
$$

(ii) $r=2$ and there exist two roots of unity $\omega_{1}, \omega_{2}$ and a constant $c$ such that for every large $n$,

$$
E_{n}(x)=c 2^{n}\left(\omega_{1}^{2^{n}}-\omega_{2}^{2^{n}}\right) x, \quad F_{n}(x)=\left(1+\omega_{1}^{2^{n}} x\right)\left(1+\omega_{2}^{2^{n}} x\right) .
$$

(iii) $r=2$ and there exist a root of unity $\omega$ and a constant $c$ such that for every large $n$,

$$
E_{n}(x)=c 4^{n} \omega^{2^{n}} x, \quad F_{n}(x)=\left(1+\omega^{2^{n}} x\right)^{2} .
$$

REMARK. It should be observed that (59) and (60) come from the case $L=1, r=2$ obtained in Theorem 8 ((59) by subtraction, as indicated at the beginning of Section 5.3, (60) by term-by-term differentiation). By contrast, (58) cannot be obtained from the case $L=r-1$. For $L=r=2$, it gives the famous series of Lucas

$$
\sum_{n=0}^{\infty} \frac{x^{2^{n}}}{1-x^{2^{n+1}}}=\frac{x}{1-x} .
$$

The general case

$$
\sum_{n=0}^{\infty} \frac{x^{r^{n}}\left(1-x^{(r-1) r^{n}}\right)}{\left(1-x^{r^{n}}\right)\left(1-x^{r^{n+1}}\right)}=\frac{x}{1-x}
$$

first appeared in Bruckman and Good [2]. Note that (58) can be obtained from Corollary $4.1 \mathrm{in} \mathrm{[7]} \mathrm{by} \mathrm{taking} c=d=r, P(x)=1-x, Q(x)=\sum_{k=0}^{r-1} x^{k}$.

Proof of Theorem 9. Assume that $\Phi_{0}(x) \in K(x)$. Then Theorem 6 applies and (49) holds. By Lemma $9, B_{n}(x) \neq 0$; by Lemma 10 , $A_{n}(x) \mid A_{n+1}\left(x^{r}\right)$; and by Lemma $9,1 \leq \operatorname{deg} A_{n} \leq 2$ for every large $n$.

First assume that $\operatorname{deg} A_{n}=1$. By Lemma $11, A_{n}(x)=1-a^{r^{n}} x$. Inserting this in (49) we obtain, as in (56),

$$
\frac{E_{n}(x)}{F_{n}(x)}=\frac{B_{n}(x) \sum_{k=0}^{r-1} a^{k r^{n}} x^{k}-B_{n+1}\left(x^{r}\right)}{1-\left(a^{r^{n}} x\right)^{r}} .
$$

As in the proof of Theorem 8 , we see that $\operatorname{deg} B_{n+1} \geq 2$ is impossible, 
therefore $B_{n}(x)=b_{n} x$ for every large $n$ and

$$
\frac{E_{n}(x)}{F_{n}(x)}=\frac{b_{n} x \sum_{k=0}^{r-1} a^{k r^{n}} x^{k}-b_{n+1} x^{r}}{1-\left(a^{r^{n}} x\right)^{r}} .
$$

By comparing the degrees of the denominators we get

$$
E_{n}(x)=b_{n} x \sum_{k=0}^{r-1} a^{k r^{n}} x^{k}-b_{n+1} x^{r}, \quad F_{n}(x)=1-\left(a^{r^{n}} x\right)^{r} .
$$

As $\operatorname{deg} E_{n}<r$, we have $b_{n+1}=a^{(r-1) r^{n}} b_{n}$ and there exists a constant $c$ such that $b_{n}=c a^{r^{n}}$. Hence

$$
E_{n}(x)=c \sum_{k=1}^{r-1} a^{k r^{n}} x^{k}
$$

and (58) holds ( $a$ must be a root of unity because of the growth conditions).

Assume now that $\operatorname{deg} A_{n}=2$. This will be more difficult. Put $A_{n+1}\left(x^{r}\right)$ $=A_{n}(x) Q_{n}(x)$ with $\operatorname{deg} Q_{n}=2 r-2$. Then (49) becomes

$$
\frac{E_{n}(x)}{F_{n}(x)}=\frac{B_{n}(x) Q_{n}(x)-B_{n+1}\left(x^{r}\right)}{A_{n+1}\left(x^{r}\right)},
$$

where we assume $\left(E_{n}, F_{n}\right)=1$ (divide $E_{n}, F_{n}$ by their greatest common divisor). Then $\operatorname{deg} E_{n}<r$ and $\operatorname{deg} F_{n} \leq r$. This implies that $A_{n+1}\left(x^{r}\right)=$ $F_{n}(x) R_{n}(x)$ with $\operatorname{deg} R_{n} \geq r$ and

$$
B_{n}(x) Q_{n}(x)-B_{n+1}\left(x^{r}\right)=E_{n}(x) R_{n}(x) .
$$

Moreover $\left(R_{n}, Q_{n}\right)=1$, otherwise $B_{n+1}\left(x^{r}\right)$ and $A_{n+1}\left(x^{r}\right)$ would have a common factor. Therefore $R_{n}(x) \mid A_{n}(x)$ and $r=2$. Since $F_{n}(0)=1$ and $A_{n+1}(0)=1$, we have $R_{n}(0)=1$ and so $R_{n}(x)=A_{n}(x), F_{n}(x)=Q_{n}(x)$, and

$$
E_{n}(x) A_{n}(x)=B_{n}(x) Q_{n}(x)-B_{n+1}\left(x^{2}\right) .
$$

As $\operatorname{deg} E_{n}(x)<r$, we have $E_{n}(x)=e_{n} x$. By putting $B_{n}(x)=x B_{n}^{*}(x)$, we obtain

$$
e_{n} A_{n}(x)=B_{n}^{*}(x) Q_{n}(x)-x B_{n+1}^{*}\left(x^{2}\right),
$$

with $\operatorname{deg} B_{n}^{*}=\operatorname{deg} B_{n}-1 \leq L-1=1$. Therefore we can put $B_{n}^{*}(x)=$ $b_{n} x+c_{n}$. We now distinguish 3 cases according to Lemma 12 .

FIRST CASE: $A_{n}(x)=\left(1-a^{2^{n}} x\right)\left(1-b^{2^{n}} x\right)$ for every large $n$. We can suppose that $a^{2^{n}} \neq \pm b^{2^{n}}$ for every large $n$, otherwise (55) holds. We also have $Q_{n}(x)=\left(1+a^{2^{n}} x\right)\left(1+b^{2^{n}} x\right)=F_{n}(x)$. Since the roots of $F_{n}(x)$ must satisfy the same growth condition as the coefficients of $F_{n}(x)$ (see [12, Lemma 1.5.4]), $a$ and $b$ are roots of unity. As the terms of degree 3 
must vanish on the right-hand side of (62), we have $b_{n+1}=(a b)^{2^{n}} b_{n}$, which implies

$$
b_{n}=c(a b)^{2^{n}} .
$$

By taking $x=a^{-2^{n}}$ and $x=b^{-2^{n}}$ in (62), we get

$$
\begin{aligned}
& 0=\left(b_{n} a^{-2^{n}}+c_{n}\right) 2\left(1+b^{2^{n}} a^{-2^{n}}\right)-a^{-2^{n}}\left(b_{n+1} a^{-2^{n+1}}+c_{n+1}\right), \\
& 0=\left(b_{n} b^{-2^{n}}+c_{n}\right) 2\left(1+a^{2^{n}} b^{-2^{n}}\right)-b^{-2^{n}}\left(b_{n+1} b^{-2^{n+1}}+c_{n+1}\right) .
\end{aligned}
$$

Using (63) yields

$$
\begin{aligned}
& 0=\left(c b^{2^{n}}+c_{n}\right) 2\left(a^{2^{n}}+b^{2^{n}}\right)-\left(c b^{2^{n+1}}+c_{n+1}\right), \\
& 0=\left(c a^{2^{n}}+c_{n}\right) 2\left(a^{2^{n}}+b^{2^{n}}\right)-\left(c a^{2^{n+1}}+c_{n+1}\right) .
\end{aligned}
$$

By subtracting these two equalities, we get $c=0$ and therefore $b_{n}=0$ for every large $n$. By (64) we now have $c_{n+1}=2\left(a^{2^{n}}+b^{2^{n}}\right) c_{n}$. Therefore there exists $c$ such that $c_{n}=c 2^{n}\left(a^{2^{n}}-b^{2^{n}}\right)$. By taking $x=0$ in (62), we see that $e_{n}=c_{n}$ and (59) holds.

According to Lemma 12, we can now assume that (55) is satisfied, that is, $A_{n}(x)=1-a^{2^{n+1}} x^{2}$ or $A_{n}(x)=\left(1-a^{2^{n}} x\right)^{2}$.

SECOND CASE: There exists $n$ such that $A_{n}(x)=1-a^{2^{n+1}} x^{2}$. Then $A_{n+1}(x)=\left(1-a^{2^{n+1}} x\right)^{2}$ is impossible, because in this case $Q_{n}(x)=(1-$ $a^{2^{n+1}} x^{2}$ ) would not be prime to $R_{n}(x)=A_{n}(x)$. Therefore $A_{n}(x)=1-$ $a^{2^{n+1}} x^{2}$ for every large $n$, and $Q_{n}(x)=1+a^{2^{n+1}} x^{2}$. As the terms of degree 3 must vanish on the right-hand side of (62), we have $b_{n+1}=a^{2^{n+1}} b_{n}$, which implies $b_{n}=c a^{2^{n+1}}$. By taking $x=a^{-2^{n}}$ and $x=-a^{-2^{n}}$ in (62), we get

$$
\begin{aligned}
& 0=\left(b_{n} a^{-2^{n}}+c_{n}\right) 2-a^{-2^{n}}\left(b_{n+1} a^{-2^{n+1}}+c_{n+1}\right), \\
& 0=\left(-b_{n} a^{-2^{n}}+c_{n}\right) 2+a^{-2^{n}}\left(-b_{n+1} a^{-2^{n+1}}+c_{n+1}\right) .
\end{aligned}
$$

By adding these two equalities, we get $2 c_{n}=a^{-2^{n}} b_{n+1} a^{-2^{n+1}}$, that is, $c_{n}=$ $c a^{2^{n}} / 2$. If we subtract them, we obtain $c_{n+1}=2 b_{n}=2 c a^{2^{n+1}}$. Hence $c=0$, a contradiction because $E_{n}(x) \neq 0$ for infinitely many $n$.

ThiRd CASE: For every large $n, A_{n}(x)=\left(1-a^{2^{n}} x\right)^{2}$. Then $Q_{n}(x)=$ $F_{n}(x)=\left(1+a^{2^{n}} x\right)^{2}$. As before, we have $b_{n+1}=a^{2^{n+1}} b_{n}$, because the righthand side of (62) must have degree 2. Therefore $b_{n}=c a^{2^{n+1}}$. Replacing $x$ by $a^{-2^{n}}$ in (62), we obtain

$$
B_{n+1}^{*}\left(a^{-2^{n+1}}\right)=4 a^{2^{n}} B_{n}^{*}\left(a^{-2^{n}}\right) .
$$

Now differentiating (62), we get

$$
\begin{aligned}
-2 e_{n} a^{2^{n}}\left(1-a^{2^{n}} x\right)= & b_{n}\left(1+a^{2^{n}} x\right)^{2}+2 a^{2^{n}} B_{n}^{*}(x)\left(1+a^{2^{n}} x\right) \\
& -B_{n+1}^{*}\left(x^{2}\right)-2 b_{n+1} x^{2} .
\end{aligned}
$$


If we replace $x$ by $a^{-2^{n}}$ and use (68), we obtain $b_{n+1}=2 a^{2^{n+1}} b_{n}$, whence $c a^{2^{n+2}}=2 c a^{2^{n+2}}$ and $c=0, b_{n}=0$. From (68) we get $c_{n+1}=4 a^{2^{n}} c_{n}$, that is, $c_{n}=c 4^{n} a^{2^{n}}$. For $x=0$ in (62), we see that $e_{n}=c_{n}$ and this is (60). The proof of Theorem 9 is complete.

5.4. Examples involving Fibonacci and Lucas numbers. Let $\alpha=$ $(1-\sqrt{5}) / 2$ and $\beta=(1+\sqrt{5}) / 2$. Then the $n$th Fibonacci number $F_{n}$ and $n$th Lucas number $L_{n}$ are written as

$$
\begin{aligned}
& F_{n}=\frac{\alpha^{n}-\beta^{n}}{\alpha-\beta}=\frac{\alpha^{n}-(-1)^{n} \alpha^{-n}}{\alpha-\beta}, \\
& L_{n}=\alpha^{n}+\beta^{n}=\alpha^{n}+(-1)^{n} \alpha^{-n} .
\end{aligned}
$$

Let $\left\{a_{k}\right\}_{k \geq 0}$ and $\left\{b_{k}\right\}_{k \geq 0}$ be sequences in $K$ and $O_{K}$ respectively. Then

$$
\begin{aligned}
& \sum_{k=1}^{\infty} \frac{a_{k}}{F_{2^{k}}+b_{k}}=(\beta-\alpha) \sum_{k=1}^{\infty} \frac{a_{k} \alpha^{2^{k}}}{1+(\beta-\alpha) b_{k} \alpha^{2^{k}}-\left(\alpha^{\left.2^{k}\right)^{2}}\right.} \\
& \sum_{k=1}^{\infty} \frac{a_{k}}{L_{2^{k}}+b_{k}}=\sum_{k=1}^{\infty} \frac{a_{k} \alpha^{2^{k}}}{1+b_{k} \alpha^{2^{k}}+\left(\alpha^{2^{k}}\right)^{2}} .
\end{aligned}
$$

Mignotte [11] proved that $\sum_{k=0}^{\infty} 1 /\left(k ! F_{2^{k}}\right)$ is transcendental by using Schmidt's theorem on approximations of an algebraic number by algebraic numbers. Later Mahler [10] proved it without using Schmidt's theorem and Loxton and van der Poorten [8] generalized Mahler's method. Becker and Töpfer [1] and Nishioka [13] studied the arithmetical nature of the series (69) and (70) when $b_{k}=0$ for every $k,\left\{a_{k}\right\}$ is a periodic sequence and a linear recurrence sequence of algebraic numbers respectively. Duverney, Kanoko and Tanaka [6] studied the case $b_{k}=b$ for every $k$ and $\left\{a_{k}\right\}$ is a linear recurrence sequence of algebraic numbers.

We have the following:

THEOREM 10. Assume that all $a_{k}$ and $b_{k}$ belong to a fixed algebraic number field $K$, that $\log \left\|a_{k}\right\|, \log \left\|b_{k}\right\|=o\left(2^{k}\right)$ and that $a_{k} \neq 0$ for infinitely many $k$. Let

$$
\Phi_{0}(x)=\sum_{k=0}^{\infty} \frac{a_{k} x^{2^{k}}}{1+(\beta-\alpha) b_{k} x^{2^{k}}-x^{2^{k+1}}} .
$$

If $\Phi_{0}(x)$ is a rational function, then there exist $N \in \mathbb{N}$ and $a \in K$ such that $b_{k}=0$ and $a_{k}=a$ for every $k \geq N$.

In particular, $\sum_{k=1}^{\infty} a_{k} /\left(F_{2^{k}}+b_{k}\right)$ is algebraic if and only if $a_{k}=a$ and $b_{k}=0$ for every $k \geq N$.

Proof. Assume that $\Phi_{0}(x) \in K(x)$. We have $F_{k}(x)=1+(\beta-\alpha) b_{k} x-x^{2}$. Therefore $F_{k}$ is not a square and (60) is impossible. Moreover, if (59) holds, then $\left(\omega_{1} \omega_{2}\right)^{2^{k}}=-1$ for every $k$, which is impossible. Therefore (58) holds 
with $r=2, \omega^{2^{k}}=1, b_{k}=0$ for every large $k, E_{k}(x)=c x$ and Theorem 10 is proved.

THEOREM 11. Assume that all $a_{k}$ and $b_{k}$ belong to a fixed algebraic number field $K$, that $\log \left\|a_{k}\right\|, \log \left\|b_{k}\right\|=o\left(2^{k}\right)$ and that $a_{k} \neq 0$ for infinitely many $k$. Let

$$
\Phi_{0}(x)=\sum_{k=0}^{\infty} \frac{a_{k} x^{2^{k}}}{1+b_{k} x^{2^{k}}+x^{2^{k+1}}} .
$$

If $\Phi_{0}(x)$ is a rational function, then one of the following two conditions is satisfied.

(i) There exist $N \in \mathbb{N}$ and $a \in K$ such that $b_{k}=2$ and $a_{k}=a 4^{k}$ for every $k \geq N$.

(ii) There exist a constant $a, p, q \in \mathbb{N}, q \neq 0$, and $N \in \mathbb{N}$ such that $b_{k}=2 \cos \left(2^{k} \cdot \frac{p}{q} \pi\right), a_{k}=a 2^{k} \sin \left(2^{k} \cdot \frac{p}{q} \pi\right)$ for every $k \geq N$. holds.

In particular, $\sum_{k=1}^{\infty} a_{k} /\left(L_{2^{k}}+b_{k}\right)$ is algebraic if and only if (i) or (ii)

Proof. Assume that $\Phi_{0}(x) \in K(x)$. Here we have $F_{k}(x)=1+b_{k} x+x^{2}$. Therefore (58) is impossible. If (60) holds, we have $\omega^{2^{k}}=1$ for every large $k$, and $b_{k}=2$. Therefore $E_{k}(x)=c 4^{k} x$, and (i) holds. If (59) holds, we have $\omega_{1}^{2^{k}} \omega_{2}^{2^{k}}=1$ for every $k \geq N$. Put $\omega_{1}^{2^{N}}=\exp \left(2 i \pi p / q_{0}\right)$, then $\omega_{2}^{2^{N}}=$ $\exp \left(-2 i \pi p / q_{0}\right)$ and for $k \geq N$,

$$
\omega_{1}^{2^{k}}=\exp \left(\frac{2 i \pi p}{q_{0} 2^{N}} 2^{k}\right)=\exp \left(\frac{i \pi p}{q} 2^{k}\right), \quad \omega_{2}^{2^{k}}=\exp \left(-\frac{i \pi p}{q} 2^{k}\right) .
$$

Therefore

$$
\begin{aligned}
& b_{k}=\omega_{1}^{2^{k}}+\omega_{2}^{2^{k}}=2 \cos \left(2^{k} \cdot \frac{p}{q} \pi\right), \\
& a_{k}=c 2^{k}\left(\omega_{1}^{2^{k}}-\omega_{2}^{2^{k}}\right)=a 2^{k} \sin \left(2^{k} \cdot \frac{p}{q} \pi\right) .
\end{aligned}
$$

This completes the proof.

COROLlary. Assume that there exist infinitely many $k$ such that $a_{k} \neq 0$, and $\log \left\|a_{k}\right\|, \log \left\|b_{k}\right\|=o\left(2^{k}\right)$. If $\sum_{k=1}^{\infty} a_{k} /\left(L_{2^{k}}+b_{k}\right)$ is algebraic, then $\left\{b_{k}\right\}$ is eventually periodic, $\left|b_{k}\right| \leq 2$ and $a_{k+1}=2 a_{k} b_{k}$ for every large $k$.

EXAMPLE 2. Under the assumptions of Theorem 11, $\sum_{k=1}^{\infty} a_{k} / L_{2^{k}}$ is transcendental. Moreover if $\left|b_{k}\right|>2$ for infinitely many $k$, then $\sum_{k=1}^{\infty} a_{k} /\left(L_{2^{k}}+b_{k}\right)$ is transcendental. 


\section{References}

[1] P.-G. Becker and T. Töpfer, Transcendency results for sums of reciprocals of linear recurrences, Math. Nachr. 168 (1994), 5-12.

[2] P. S. Bruckman and I. J. Good, A generalization of a series of de Morgan, with applications of Fibonacci type, Fibonacci Quart. 14 (1976), 193-196.

[3] D. Duverney, Théorie des Nombres, Dunod, Paris, 1998.

[4] - Irrationality of fast converging series of rational numbers, J. Math. Sci. Univ. Tokyo 8 (2001), 275-316.

[5] - Transcendence of a fast converging series of rational numbers, Math. Proc. Cambridge Philos. Soc. 130 (2001), 193-207.

[6] D. Duverney, T. Kanoko and T. Tanaka, Transcendence of certain reciprocal sums of linear recurrences, Monatsh. Math. 137 (2002), 115-128.

[7] D. Duverney and I. Shiokawa, On series involving Fibonacci and Lucas numbers I, preprint.

[8] J. H. Loxton and A. J. van der Poorten, Arithmetic properties of certain functions in several variables III, Bull. Austral. Math. Soc. 16 (1977), 15-47.

[9] E. Lucas, Théorie des fonctions numériques simplement périodiques, Amer. J. Math. 1 (1878), 184-240 and 289-321.

[10] K. Mahler, On the transcendency of the solutions of a special class of functional equations, Bull. Austral. Math. Soc. 13 (1975), 389-410.

[11] M. Mignotte, Quelques problèmes d'effectivité en théorie des nombres, DSc thèse, Univ. de Paris XIII, 1974.

[12] K. Nishioka, Mahler Functions and Transcendence, Lecture Notes in Math. 1631, Springer, 1996.

[13] - Algebraic independence of reciprocal sums of binary recurrences, Monatsh. Math. 123 (1997), 135-148.

Appartement 3501

13 , rue de Roubaix

59800 Lille, France

E-mail: dduverney@nordnet.fr
Mathematics, Hiyoshi Campus Keio University 4-1-1 Hiyoshi, Kohoku-ku Yokohama 223-8521, Japan

E-mail: kumi-nis@jcom.home.ne.jp

Received on 30.7.2002

and in revised form on 15.1.2003 